\title{
la empresa familiar desde el sentir y el pensar: Un modelo de gedtión del conocimiento
}

\section{The family company from fecling and thinking: a knowledge management model}

Lissette Hernández Fernández ${ }^{1}$

Rafael Portillo Medina ${ }^{2}$

Ana Hernández Chacín ${ }^{3}$ Jenny Romero Borré ${ }^{4}$

1 Universidad de la Costa (CUC). Barranquilla-Colombia. lhernand31cuc.edu.co; lissettehf@yahoo.es - https://orcid.org/0000-0003-0839-6057

2 Universidad de la Costa (CUC). Barranquilla-Colombia. rportill3@cuc.edu.co; rportillomedina@gmail.com - https://orcid.org/0000-0002-15220562

3 Universidad de la Costa (CUC). Barranquilla-Colombia. ahernand48@cuc.edu.co; hernandezanaemilia@gmail.com - https://orcid.org/0000-0002 $-4246-0713$

4 Universidad de la Costa (CUC). Barranquilla-Colombia. jromero58@cuc.edu.co; jennyfrb@yahoo.com - https://orcid.org/0000-0002-0457-6467 


\section{Gerencia de las organizaciones.}

Un enfoque empresarial

\section{RESUMEN}

La empresa familiar es el tipo de organización con mayor presencia en el mundo, caracterizada por conjugar dos sistemas con lógicas distintas: la familia y la empresa. El primero es emocional, actúa en pro del bienestar del grupo familiar; y el segundo responde a criterios de racionalidad económica. Estas organizaciones actualmente enfrentan importantes retos, uno de carácter externo, vinculado al proceso de globalización, el cual exige competitividad; y otro derivado de su dinámica interna, lograr el balance entre los intereses y objetivos de la empresa y la familia. La propuesta de un modelo de gestión del conocimiento en la empresa familiar, objeto del presente trabajo, constituye una alternativa en pro de ambos retos. El enfoque epistemológico seguido es el racionalista-deductivo, con un tipo de investigación analítica, ya que con base en la teoría y modelos que explican la dinámica de la empresa familiar se configura el modelo de gestión del conocimiento. El flujo de conocimiento entre los diferentes sistemas que constituyen la empresa familiar, y la espiral del conocimiento que se genera de sus interacciones, contribuirán a mejorar la función administración mediante el equilibrio entre lo emocional y lo racional, redundando en el fortalecimiento competitivo de la organización.

Palabras clave: empresa familiar, gestión del conocimiento, profesionalización empresarial, emocionalidad, racionalidad.

\section{ABSTRACT}

The family business is the type of organization with the greatest presence in the world, characterized by combining two systems with different logics: the family and the company. The first, is 
emotional, acts for the well-being of the family group; and the second, responds to criteria of economic rationality. These organizations are currently facing significant challenges, one of an external nature, linked to the globalization process, which requires competitiveness; and another derived from its internal dynamics, to achieve the balance between the interests and objectives of the company and the family. The proposal of a model of knowledge management in the family enterprise, object of the present work, constitutes an alternative in favor of both challenges. The epistemological approach followed is the rationalist deductive, with a type of analytical research, since based on the theory and models that explain the dynamics of family business, the knowledge management model is configured. The flow of knowledge between the different systems that constitute the family business, and the spiral of knowledge generated from their interactions, will contribute to improve the administration function through the balance between the emotional and the rational, resulting in the competitive strengthening of the organization.

Keywords: family business, knowledge management, business professionalization, emotionality, rationality.

\section{INTRODUCCIÓN}

La empresa familiar constituye uno de los pilares básicos de las economías como generadora de riqueza, dada su contribución al Producto Nacional Bruto (PNB) y el empleo en los países. Se estima que entre el $65 \%$ y el $85 \%$ de las empresas del mundo son empresas familiares; y su contribución al empleo varía según los países, generando como mínimo un $60 \%$ de los puestos de trabajo en las distintas economías (Gersick, Davis, Hampton \& 


\section{Gerencia de las organizaciones.}

Un enfoque empresarial

Lansberg, 1997). Por otra parte, en EEUU, según el ranking de la revista Fortune, el $40 \%$ de las 500 empresas más grandes es de carácter familiar (Harvard Business School Bulletin, 1990, p.78. Ginebra, 2005); mientras que en España, el Instituto de Empresa Familiar señala que el $17 \%$ de las 1.000 mayores empresas son familiares (Ruzo, Lozada y Gómez, 2007). Asimismo, diversos trabajos presentan una realidad similar en otros tantos países, con una presencia abrumadoramente mayoritaria de empresas familiares en las pequeñas y medianas empresas (cfr. Monreal y Sánchez, 2012; Corona, 2005; Gómez-Betancourt, 2006; Dodero, 2008).

Una empresa familiar no es diferente a cualquier otra organización, salvo en un aspecto muy importante, la propiedad o su control está en manos de una familia o asociación de familias, y por lo general, son los miembros de ellas los que ocupan los puestos decisivos de la compañía. Esta dimensión familiar diferencia a estas empresas de todas las demás y hace de este tipo de organización una institución emocional, de modo que el fundador puede verse confundido cuando los intereses y objetivos de la empresa y la familia, muchas veces contradictorios, se traslapan. Las normas de la familia en cuanto a selección de personal, compensación económica, valoración de los individuos y formación de los mismos, no coinciden con las normas de la empresa en general, y esto afecta inevitablemente la estructura de este tipo de organizaciones. Es un devenir permanente entre el sentir y el pensar; entre lo emocional y lo racional.

El hecho de dirigir una empresa, cuya propiedad es de la familia, en la que participan miembros de esta en su gestión y funciona- 
miento, genera los solapamientos entre los diferentes sistemas que la integran (familia-empresa-propiedad); y la confusión en la toma de decisiones, generalmente más emocionales que racionales, dados los diferentes roles que se desempeñan en la empresa. Por esa razón se afirma que la familia es, ante todo, una institución emocional. Por ello los negocios de este tipo son una mezcla de sentimientos familiares, y necesidades empresariales. Los niveles de confianza y lealtad existentes dentro de la familia afectan el desarrollo de la empresa, haciéndolas, con gran frecuencia, ineficientes en sus respuestas al mercado.

La realidad demuestra que en la empresa familiar priman, muchas veces, los compromisos familiares por sobre las exigencias del mercado y de la actividad propia de la empresa. El rigor, la reflexión en la toma de decisiones, la implantación de códigos de buen gobierno en el funcionamiento interno de las empresas familiares, sucumben con más frecuencia de la deseada al peso de los compromisos familiares.

Como respuesta a este comportamiento, múltiples autores visualizan la profesionalización de la empresa familiar como una vía para mitigar o eliminar, en algunos casos, el conflicto emocional de trabajar con la familia, y responder oportunamente a las demandas del mercado (clientes y proveedores) alcanzando mayores niveles de competitividad, (Cromie, Dunn, Sproull \& Chalmers, 2001; León-Guerrero, McCann \& Haley Jr., 1998; Astrachan y Kolenko, 1994; Aragón, Iturrioz, Aranguren y Olarte, 2005; Gersick et al., 1997; Amat, 2004; Amat, Roure, y Martínez, 2008; Tadeo Basco, 2005; y Bosch Sans, 2006).

La profesionalización de la dirección y de sus sistemas de fun- 


\section{Gerencia de las organizaciones.}

Un enfoque empresarial

cionamiento interno, así como del gobierno de la empresa, permiten resolver, por una parte, los conflictos emocionales y psicológicos derivados de las relaciones a lo interno de las empresas familiares; y por la otra, crear y/o adaptar las estructuras necesarias para dar respuestas al mercado de manera competitiva. En el caso de las primeras, es necesario trabajar sobre las estructuras de gobierno de la familia (asamblea y/o consejo de familia, protocolo familiar, plan de sucesión) y de la empresa (junta de accionistas o propietarios, consejo de administración y dirección); y las segundas, hacen referencia a las estructuras y diseños organizativos, políticas de recursos humanos y las tecnologías de información y comunicación (TIC) (Hernández, 2009).

La profesionalización empresarial se traduce en que las perso-

188 nas que integran la organización se adapten a un enfoque más empresarial que familiar. En este sentido, la gestión del conocimiento destaca como soporte de la profesionalización empresarial, ya que garantiza el flujo de conocimiento en la organización de vital relevancia para la toma de decisión, lográndose brindar respuestas oportunas a los distintos grupos de interés que en la dinámica de la empresa interactúan.

En este momento el factor de producción crítico que permite a las distintas empresas alcanzar sus objetivos es algo que gira en torno a la capacidad de esas organizaciones para captar, asimilar y distribuir el conocimiento que reside no solo en la organización, sino fundamentalmente en las personas que la integran. Con ese conocimiento se genera una nueva manera de producir, de organizar, de atender las crecientes y complejas necesidades del mercado. 


\section{CONSIDERACIONES TEÓRICAS}

El modelo de gestión del conocimiento en la empresa familiar se desarrolla a partir del denominado "modelo de los tres círculos" (Gráfico No 1), propuesto por Tagiuri y Davis (1982. En Gersick et al., 1997), al que se le introduce la variable "conocimiento".

El conocimiento es definido por Davenport y Prusak (1998) como el conjunto de saberes integrado por información, reglas, interpretaciones y conexiones puestas dentro de un contexto y una experiencia que ha sucedido dentro de una organización, bien sea de forma general o personal. El conocimiento es personal, en el sentido de que se origina y reside en las personas que lo asimilan como resultado de su propia experiencia, es decir, de su propio "hacer", ya sea físico o intelectual, y lo incorporan a su acervo personal estando "convencidos" de su significado e implicaciones,

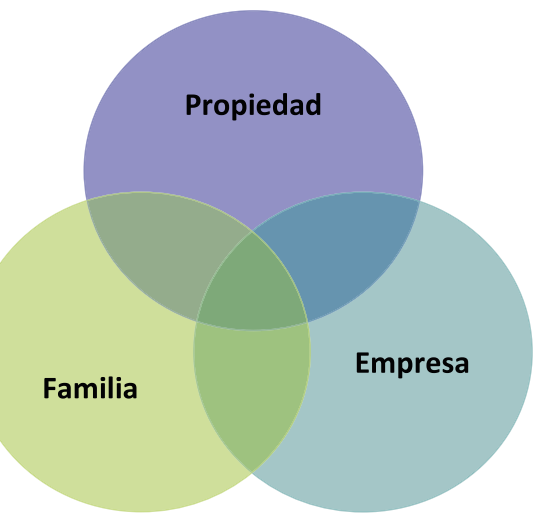

Gráfico 1

El modelo de los tres círculos Fuente: Tagiuri y Davis (1982. En Gersick et al., 1997). articulándolo como un todo organizado que da estructura y significado a sus distintos elementos.

El movimiento del conocimiento está sacudiendo los fundamentos mismos de cómo una organización se crea, desarrolla y madura, muere y se reforma; generando cambios fundamentales en la manera como se hacen negocios. 


\section{Gerencia de las organizaciones.}

Un enfoque empresarial

El conocimiento entendido de esta manera, adquiere una importancia fundamental en el funcionamiento eficiente de las organizaciones empresariales, incluyendo en estas a las "empresas familiares". Con esta finalidad es necesario distinguir entre sus dos dimensiones (cfr. Nonaka, 2000; Polanyi, 1975; Dewey, 1991):

- Explícito: Es el conocimiento que los filósofos reconocieron como el "saber qué" (knowing that); también ha sido llamado conocimiento proposicional, objetivo, formal (teórico) y sistemático. Este tipo de conocimiento pone énfasis en la capacidad de estructurar la experiencia por medio de conceptos, causas, efectos, razones, y finalmente en la prescripción de leyes científicas universales. Sus productos se definen normalmente como ideas o abstracciones. Este tipo de conocimiento no requiere de validación por medio de la experiencia personal. En otras palabras, el "saber qué" es explícito porque

190 los seres humanos pueden hablar acerca de él, poniendo en palabras las unidades de sentido que llegan a conocer o construir; v.g. especificaciones de producto, una fórmula científica o un programa de ordenador.

- Tácito o Implícito: Denominado por los filósofos "saber cómo" (knowing how); también se le conoce como conocimiento procedimental, subjetivo (intuición), práctico o personal. Este tipo de conocimiento es asociado a formas específicas de competencias (performance). El "saber cómo" es el reconocerse poseedor de la habilidad de ejecutar una acción; consiste en conocimiento técnico, esa clase de capacidades y conocimientos informales y difíciles de concretar que se suelen englobar en el término (Know-How); v.g. un artesano, después de muchos años de experiencia, adquiere muchos conocimientos, pero es incapaz de articular los principios técnicos o científicos de lo que sabe. 
- La diferenciación entre conocimiento explícito e implícito (tácito) indica las cuatro formas en que se puede crear conocimiento en una empresa: de tácito a tácito, de explícito a explícito, de tácito a explícito y de explícito a tácito (cfr. Nonaka, 2000) (ver Cuadro 1).

\section{Cuadro 1}

La espiral del conocimiento

\begin{tabular}{l|l|l}
\hline & \multicolumn{1}{c}{ A Tácito } & \multicolumn{1}{c}{ A Explícito } \\
\hline \multirow{5}{*}{ De Tácito } & $\begin{array}{l}\text { Socialización. } \\
\text { Interacción directa con el } \\
\text { mundo exterior: compartir } \\
\text { con otras personas, otras } \\
\text { culturas. Conocimiento basa- } \\
\text { do en la observación, la imita- } \\
\text { ción y la práctica. }\end{array}$ & $\begin{array}{l}\text { Externalización. } \\
\text { Diálogo: proceso de expresar } \\
\text { algo yecibir algo (retroali- } \\
\text { mentación). }\end{array}$ \\
& $\begin{array}{l}\text { Internalización. } \\
\text { Interiorizarlo: ampliar, ex- } \\
\text { tender y modificar su propio } \\
\text { conocimiento tácito. }\end{array}$ & $\begin{array}{l}\text { Combinación. } \\
\text { Recopilan e integran partes } \\
\text { separadas de conocimiento } \\
\text { para establecer nuevo cono- } \\
\text { cimiento; combinándolos. }\end{array}$ \\
\hline
\end{tabular}

Fuente: Hernández, Portillo, Hernández y Romero (2016), Adaptado de Nonaka (2000).

\section{MÉTODO}

Para la propuesta de un modelo de gestión del conocimiento en la empresa familiar, el enfoque epistemológico seguido es el racionalista-deductivo, con un tipo de investigación analítica, ya que con base en la teoría y modelos que explican la dinámica de la empresa familiar se configura el modelo de gestión del conocimiento. Con esta finalidad se realizó una revisión documental, empleándose como técnica el arqueo bibliográfico de artículos en revistas del área de las ciencias sociales que figuran en el Scimago Journal Report (SJR), que contemplan dimensiones de la dinámica de las empresas familiares, tales como, modelos explicativos, conflicto de roles, grupos de interés y gestión del conocimiento. Estas mismas dimensiones, permitieron filtrar artículos a través de google scholar, jerarquizado en función del número de citaciones. 


\section{Gerencia de las organizaciones.}

Un enfoque empresarial

El método deductivo utilizado para la construcción de un modelo de gestión del conocimiento en las empresas familiares partiendo de teorías de entrada, fue fortalecido mediante el conocimiento que los autores del presente artículo poseen derivado de la experiencia como consultores en este tipo de organizaciones, en temas relativos a la red de relaciones entre los distintos grupos de interés (stakeholders), y flujo de información y transferencia de conocimiento entre estos.

Posteriormente se solicitó a los directivos de una muestra de empresas del sector comercio, específicamente del ramo del retail, validar el modelo de gestión del conocimiento en la empresa familiar, así como la espiral del conocimiento que se gesta en este tipo de unidades económicas.

\section{MODELO DE GESTIÓN DEL CONOCIMIENTO EN LA EMPRESA FAMILIAR}

En el modelo de gestión del conocimiento en la empresa familiar, cada uno de los círculos representa una de las siguientes zonas:

- Familia (F): Se define a través de la diversidad de relaciones por consanguinidad ${ }^{5} \mathrm{y}$ afinidad ${ }^{6}$ que une a los miembros de una familia, v.g. padres, hermanos, primos y familiares políticos (suegros, yernos, cuñados, entre otros).

- Propiedad (P): Caracterizada por la estructura accionarial de la empresa, la cual puede ser familiar y no familiar. Generalmente en este tipo de empresas se establecen límites a la cesión de acciones a no familiares, ante el temor de la pérdida del

5 Unión por parentesco natural, de varias personas que descienden de una misma raíz o tronco.

6 Parentesco que a través del matrimonio se establece entre cada cónyuge y los familiares del otro. 
poder sobre la misma. El carácter de la intervención de los accionistas podrá ser pasiva o activa, esta última estará en función de su participación en las actividades de la empresa, bien sea como empleado o directivo de la misma.

- Empresa o Negocio (E): Hace referencia al conglomerado de individuos (recurso humano) cumpliendo actividades como empleado o directivo.

- Conocimiento (Cn): Integrado por el conjunto de saberes tanto explícitos como implícitos (tácitos). El primero está referido a la preparación o formación en carreras, profesiones y especializaciones puntales para la empresa (CnE); y el segundo trata del conocimiento derivado de la práctica, de la experiencia, manifestado no solo a través de las habilidades para ejecutar una acción, sino también por la comprensión de la forma de comportarse (reaccionar) la organización, es decir, el conocimiento de su cultura (CnI).

Es importante señalar que esta última dimensión del conocimiento no es exclusiva del círculo del conocimiento, es decir, que coexiste en las otras áreas; por su carácter individualista y personal está presente en todas las etapas de la vida del individuo, vinculándolas a diferentes contextos.

En el modelo las personas que interactúan en la empresa familiar tendrán conocimiento tácito - derivado tanto de su propio aprendizaje, como el resultante del proceso de "socialización" con sus pares- de las áreas en las cuales se encuentran ubicados: familia, propiedad y empresa. Este conocimiento se originará de su actividad en la empresa familiar como miembro de la familia, como accionista o empleado, además del logrado mediante la participación en los Consejos de Familia, Juntas de Accionistas, Consejo de Administración y reuniones de empleados. 


\section{Gerencia de las organizaciones.}

Un enfoque empresarial

El carácter "evolutivo" es una característica de este modelo, confiriéndole la propiedad de dinamismo, en contraposición con lo estático, muy acorde con la definición de conocimiento, el cual se desarrolla y avanza, pudiendo reflejarse su evolución mediante la generación de la espiral del conocimiento en la empresa familiar mediante la transmisión del conocimiento explícito (cuya presencia significa la concreción del proceso de profesionalización de los recursos humanos), el conocimiento implícito o tácito, y la interacción entre ambos (socialización, internalización, externalización y combinación) (Cuadro 1).

En la conjunción de ambos tipos de conocimiento es donde se encuentra, como señala Nonaka (2000), "la verdadera esencia de la creación de conocimiento", porque es donde se combina la teoría con la experiencia y la práctica, potenciándose la capacidad inno-

194 vativa. En los otros estadios, de implícito a implícito y de explícito a explícito, aun cuando existe un fortalecimiento o ampliación del conocimiento, "la creación del mismo es bastante limitada" (Nonaka, 2000, p.31) dado que no se produce un enriquecimiento mediante la contrastación de la teoría con la práctica.

De esto se deriva que las áreas en el modelo donde estén presentes ambas dimensiones del conocimiento se consideren como "óptimas", en términos de garantizar la operatividad y gestión de la empresa de manera eficiente.

A través del Gráfico 2 se pueden visualizar las diferentes áreas que se derivan de la yuxtaposición de los cuatro círculos o zonas: - Área 1: Hace referencia a aquellas personas que son miembros de la familia (F): padres, hermanos, primos, suegros, yernos, cuñados, entre otros, y poseen conocimiento implícito 
(CnI) derivado de la experiencia personal dentro de la familia, o mediante el intercambio de información en el seno del grupo familiar.

- Área 2: Reúne al conjunto de personas que son propietarios (P) no familiares, es decir, que tienen participación en el capital de la empresa. Generalmente, las empresas familiares establecen límites a la propiedad no familiar. Sin embargo, este grupo puede comprender tanto personas de confianza de la empresa, especialmente amigos de la familia dueña del negocio, así como personas que han sido familiares políticos, pero por divorcio o separación han dejado de ser familiares; y además poseen conocimiento implícito (CnI) originado de su experiencia como propietario del capital mediante su participación en la Junta de Accionistas, entre otras.

- Área 3: Contempla el recurso humano no familiar que se desempeña como empleado, carente de formación profesional (conocimiento explícito); cuya fortaleza es la posesión de un invalorable conocimiento (tácito) sobre una actividad particular de la empresa (CnI). Un ejemplo de este tipo de empleado puede ser un obrero u operador de una maquinaría.

- Área 4: Está representada por aquellas personas que tienen participación en el capital (P) y son miembros de la familia (F). En esta categoría figuran los familiares directos (padres, hermanos, primos, entre otros), cuya actividad laboral es desempeñada fuera del negocio de la familia; o familiares políticos (yernos) que por razón de matrimonio pasan a formar parte de la estructura accionarial, aun cuando no participan directamente en la vida de la empresa. Además, cuentan con un conocimiento implícito ( $\mathrm{CnI}$ ) obtenido de la experiencia acerca del funcionamiento de la familia y la propiedad, fortalecida a 


\section{Gerencia de las organizaciones.}

Un enfoque empresarial

través de los Consejos de Familia y Juntas de Accionistas.

- Área 5: Está constituida por los propietarios (P) no familiares que laboran como empleados y poseen el conocimiento derivado de la práctica o experiencia ( $\mathrm{CnI})$ en una actividad dentro de la empresa familiar. Entre estos destacan los empleados de confianza que han recibido una participación minoritaria en el capital para retenerlos dentro de la compañía y/o recompensar su lealtad, y los yernos que por divorcio dejan de ser familia, mantienen su participación accionaria y su cargo como empleado del negocio.

- Área 6: Hace referencia a los familiares que cumplen una actividad dentro de la empresa y poseen conocimiento $(\mathrm{Cn}=\mathrm{CnE}$ + CnI). Por tanto, contempla tanto a familiares directos (lazos consanguíneos) como políticos (lazos por afinidad); v.g. padres, hermanos, yernos, cuñados, entre otros, que se desempeñan bien como empleado, o como directivo, dado su nivel de profesionalización (CnE) como por su experiencia personal dentro de la familia y la empresa (CnI), otorgándole esta última el conocimiento de ciertos aspectos y actividades propias de dicha unidad económica.

- Área 7: En esta confluyen los nexos de familiaridad con propiedad (control de acciones), y participación en la dirección y operación del negocio familiar, la cual se ejecutará eficientemente dado el don del conocimiento $(\mathrm{Cn})$, no solo a través de la profesionalización en áreas administrativas y gerenciales, entre otras, sino también mediante el conocimiento otorgado por la praxis de gestión en la empresa familiar. Es en esta zona donde se ubican los familiares directos y políticos que mayor importancia y valor le dan a la empresa familiar, ya que en ella concentran su riqueza o patrimonio, además de 
ser la fuente de empleo de él y sus descendientes. Esta área es considerada, dadas las características de las personas que en ella se ubican: familiar, propietario, empleado con conocimiento (Cn), como el área «óptima», por el fuerte compromiso que se genera en sus miembros en pro de la consecución del mejoramiento de los procesos de la empresa, garantizando la gestión de manera eficiente, en el cual juega un papel fundamental el uso del conocimiento (Cn) presente, así como su constante avance y perfeccionamiento, tanto en el área de la profesionalización como en el desarrollo y fortalecimiento de actividades y labores producto de la experiencia.

- Área 8: Constituida por aquellas personas que tienen participación en el capital (propietarios) con conocimiento explícito, es decir, profesionalizados. Representado bien sea por amigos de la familia, exesposos(as) o por exfamiliares políticos (yernos, cuñados, entre otros) que mantienen participación accionaria de la empresa, y además poseen conocimiento explícito (están capacitados, profesionalizados), que solo es puesto a disposición de la organización a través de la intervención en la Junta de Accionistas, ya que no laboran en la empresa familiar.

- Área 9: las personas establecidas en este sector reúnen similares características que las que se ubican en el área 8, con la única diferencia que los propietarios son miembros de la familia; por tanto la transmisión del conocimiento y aportes en términos del mejoramiento de los procesos en la empresa familiar se ejecutará tanto por la vía de la Junta de Accionistas, como por la vía de los Consejos de Familia y el intercambio personal (diálogo) entre miembros de la familia, lo que se traduce en un mayor nivel de influencia en las decisiones de 


\section{Gerencia de las organizaciones.}

Un enfoque empresarial

la empresa por parte de estas personas en comparación con las del área anterior.

- Área 10: Definida por aquellas personas que son propietarias (participan en el capital), comparten la responsabilidad laboral, bien sea como gerente, directivo o empleado y poseen conocimiento explícito en el área gerencial administrativa, dirección de empresas, etc., así como el conocimiento acerca de los procesos y actividades de la empresa (conocimiento implícito). Ambos fundamentales en la orientación hacia el mercado de la empresa familiar de manera competitiva. Las personas que integran esta área son individuos que han adquirido una participación minoritaria basado en la confianza que les profesan los miembros de la familia propietaria de la empresa, o como recompensa por su lealtad como empleado; así como también pueden formar parte de ella los exfamiliares políticos que aún mantienen una cuota de acciones.

- Área 11: Conformada por miembros de la familia, bien sea por la línea por consanguinidad o afinidad, que por decisión propia o por decisiones familiares no han tenido opción de participar en la estructura accionaria, y han cumplido o culminado con su etapa de formación académica (CnE), laborando y desarrollándose como profesionales en otras instituciones u organizaciones. Entre estos destacan los hijos que por exigencia propia o por decisión de los padres les corresponde como herencia otras propiedades, o el caso de las parejas que deciden, previa a la unión conyugal, separar sus bienes (firman capitulaciones).

- Área 12: Representada por empleados (gerentes, cuadros intermedios y el resto del personal) con conocimiento $(\mathrm{Cn})$ en el ámbito académico (profesionalizados) como en el dominio 
de la empresa (actividades, procesos, forma de comportarse, etc.). Son estos empleados, en función de la responsabilidad y lealtad con que ejecuten sus funciones, los candidatos potenciales a constituirse en propietarios, de acordarse la decisión de negociar acciones.

- Área 13: Compuesta por un conjunto muy heterogéneo de personas con conocimiento explícito, es decir, con formación universitaria y/o técnica, que no son propietarios, empleados, ni miembros de la familia dueña de la empresa, pudiendo comprender a clientes, proveedores, personal ya jubilado de la compañía, y profesionales externos de la empresa (consultores y "banco de elegibles" para iniciar su carrera como empleado de la empresa familiar).

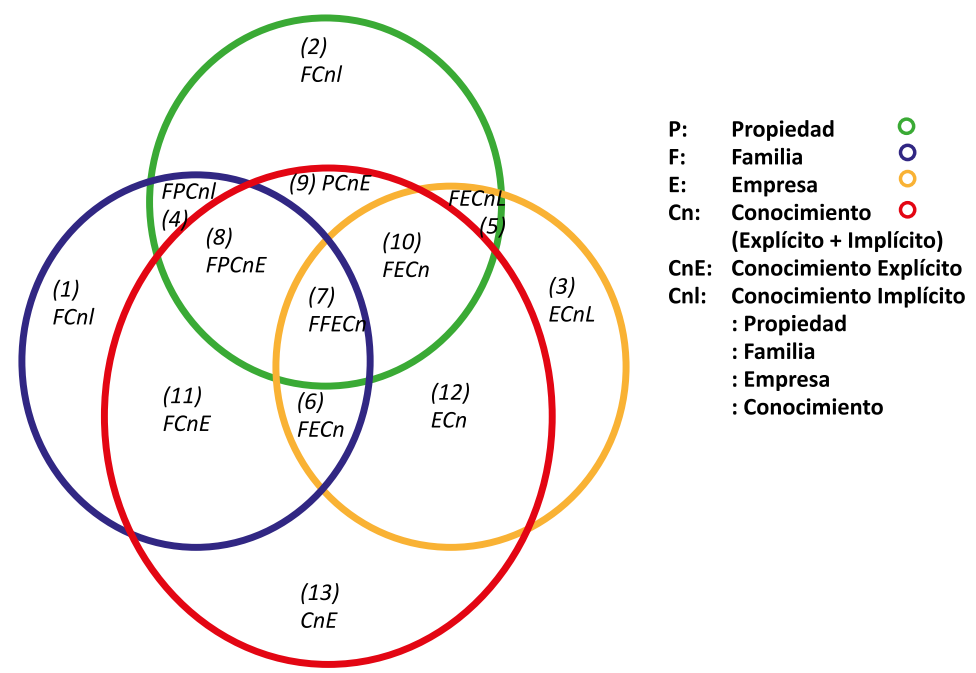

Gráfico 2

Modelo de gestión del conocimiento en la empresa familiar Fuente: Adaptado por los autores. Según modelo de los tres circulos de Tagluri y Davis (1992. en Gersick et. al. 1997)

A través de la definición e ilustración de cada una de las áreas del "Modelo de gestión del conocimiento de la empresa fami- 


\section{Gerencia de las organizaciones.}

Un enfoque empresarial

liar" (Gráfico 2) se observa que el conocimiento implícito o tácito (CnI) se presenta en las áreas $1,2,3,4$, y 5, otorgándole a los individuos los saberes derivados de la experiencia en las zonas donde se ubican (familia, empresa y propiedad); el conocimiento explícito (CnE) está expresado a través de aquellas áreas de la esfera del conocimiento que no tocan el círculo de la empresa, v.g. áreas 8, 9, 11 y 13; y solo transita hacia el estadio del conocimiento pleno $(\mathrm{Cn})$ donde se conjuga la preparación o formación del recurso humano ( $\mathrm{CnE}$ ) con el conocimiento en los diversos aspectos y actividades de la empresa (CnI) en las áreas 6, 7, 10 y 12.

Se reconoce que este último estadio es el "ideal", es la etapa hacia la cual se debe dirigir la empresa familiar para el logro de su eficiencia, lo que contribuirá a su permanencia en el mercado mediante el fortalecimiento de sus ventajas competitivas. Sin embargo, se está consciente de que es un proceso que necesita tiempo, razón por la cual, mientras se transita en la consecución de la misma, en las áreas $6,7,10$ y 12 se pueden ubicar personas (familiares, empleados, propietarios y no propietarios, y propietarios y empleados no familiares, respectivamente) en proceso de profesionalización; así como también individuos, particularmente en las áreas 6 y 7, carentes de conocimiento explícito por constituir la generación fundadora de la empresa familiar, en la cual no tenía un lugar prominente la formación y la capacitación del recurso humano. Casos como este se visualizan en aquellas empresas que actualmente se encuentran entre la segunda y tercera generación; pero que sin embargo comprenden el proceso, y aceptan y asumen la formación de la generación de relevo, no solo de aquellos a quienes se les entregará la batuta, sino de 
todo el personal, llegando muchas veces a participar los mismos fundadores en cursos de actualización ${ }^{7}$.

Por tanto, la espiral del conocimiento en este modelo se cumple de la siguiente forma:

- En las áreas 8, 9, 11 y 13 se transita de conocimiento explícito a explícito, es decir, se recopilan e integran conocimientos teóricos,

- En las áreas 1, 2, 3, 4 y 5 se lleva a efecto la transmisión de conocimiento tácito, mediante la socialización de diferentes experiencias, $\mathrm{y}$

- En las áreas 6, 7, 10 y 12 es donde se efectúa el verdadero proceso de creación de nuevo conocimiento, porque es donde se transfiere de conocimiento explícito a tácito y viceversa.

\section{CONCLUSIÓN}

El modelo de gestión del conocimiento en la empresa familiar tiene como objetivos, por una parte, la mejora de la competitividad empresarial, ya que a través de la presencia del conocimiento en el proceso productivo o en la gestión y/o administración, se estará incorporando valor agregado a los productos y/o servicios que se ofrecen. Mientras más profesionalizada esté la empresa (el recurso humano, la estructura organizativa y tecnológica) para dar respuesta a las exigencias del mercado, más posibilidades tendrá de librar la batalla competitiva.

Por otra parte, este modelo coadyuvará a superar los desbalan-

\footnotetext{
7 Aduciendo "el conocimiento" como característica fundamental de la eficiencia empresarial, lo que a su vez garantizará su permanencia en el mercado mediante el desarrollo y/o creación de ventajas competitivas (lo que Krugman (1994) y otros postulantes de las teorías sobre Comercio Internacional denominan "Ventajas Competitivas Dinámicas").
} 


\section{Gerencia de las organizaciones.}

Un enfoque empresarial

ces o diferencias entre los intereses de los sistemas familia y empresa, ya que a través de los flujos de información estarán en interacción los distintos grupos de interés de la organización, conociendo y poniendo bajo reflexión las necesidades de ambos sistemas, tan importantes para la toma de decisión que la conduzca hacia su permanencia o sustentabilidad. ${ }^{8}$

\section{REFERENCIAS BIBLIOGRÁFICAS}

Amat, J. (2004). La continuidad de la empresa familiar. España: Ediciones Gestión 2000, S.A.

Amat, J., Roure, J. y Martínez, J. (2008). Transformarse o desaparecer. Estrategias de la empresa familiar para competir en el siglo XXI. España: Editorial Deusto.

Aragón, C., Iturrioz, C., Aranguren, M. y Olarte, F. (2005). La empresa familiar en Gipuzkoa. España: Cámara de Gipuzkoa.

202 Astrachan, J. y Kolenko, T. (1994). Un aspecto ignorado que explica el éxito de la empresa familiar: Las prácticas de recursos humanos. Family Business Review, 7(3). Family Firm Institute (FFI), 251-262.

Bosch Sans, V. (2006). Alta dirección y empresa familiar. Lupa Empresarial On Line. Asociación Catalana de Empresa Familiar. CEIPA. Recuperado en: http://www. ceipa.edu.co/ceipa/ subsitios/lupa_empresarial/ediciones_online/alta_direccion. pdf

Corona, J. (2005). Manual de la empresa familiar. España: Ediciones Deusto.

Cromie, S., Dunn, B., Sproull, A. \& Chalmers, D. (2001). Small Fir-

8 La brecha en la preparación de los padres respecto de la de los hijos, se ha reducido notablemente y lo será también muy pronto entre abuelos y nietos; transformaciones que tienen su relevancia en la gestión de la empresa familiar. Padres e hijos estudiados implica mayor posibilidad de entendimiento en el ámbito de los negocios (Lozano, S/A, p.7). 
ms with a Family Focus in the Scottish Highlands and Islands. The Irish Journal of Management, 22(2). 45-66. Irlanda.

Davenport, T. H., \& Prusak, L. (1998). Working Knowledge: How Organizations Manage What They Know. Estados Unidos: Harvard Business Press.

Dewey, J. (1991). How We Think. Buffalo, New York: Prometheus Books.

Dodero, S. (2008). El secreto de las empresas familiares exitosas. Argentina: Editorial El Ateneo.

Gersick, K. E., Davis, J. A., Hampton, M. M. \& Lansberg, I. (1997). Empresas familiares: Generación a generación. México: McGraw-Hill.

Ginebra, J. (2005). Las empresas familiares. Su dirección y su continuidad. México: Panorama Editorial S.A.

Gómez-Betancourt, G. (2006). ¿Son iguales todas las empresas familiares? Caminos por recorrer. Colombia: Grupo Editorial Norma.

Hernández, F. L. (2009). Construcción teórica de la profesionalización empresarial en organizaciones familiares. Cadenas de hipermercados y supermercados familiares en Venezuela (Tesis Doctoral) Doctorado en Ciencias Humanas. Facultad de Humanidades y Educación. Maracaibo, Venezuela: Universidad del Zulia.

Krugman, P. (1994). Competitiveness: A Dangerous Obsession. Foreign Affairs, 73(2), 28-44.

León-Guerrero, A., McCann, J. \& Haley Jr. J. (1998). A Study of Practice Utilization in Family Businesses. Family Business Review, 11(2), 107-120.

Lozano, M. (S/A). La empresa familiar: Factores contemporáneos y su influencia en la gestión. En: http://lanic.utexas.edu/ pyme/esp/publicaciones/biblioteca/itcr/familiar.html 


\section{Gerencia de las organizaciones. \\ Un enfoque empresarial}

Monreal, J. y Sánchez, G. (Coord.). (2012). El éxito de la empresa familiar. La relación entre negocio y familia. España: Editorial Aranzadi, S.A.

Nonaka, I. (2000). La empresa creadora de conocimiento. Gestión del Conocimiento. Harvard Business Review. España: Ediciones Deusto S.A.

Polanyi, M. \& Prosch, H. (1975). Meaning. Chicago: University of Chicago Press.

Ruzo, E., Lozada F. y Gómez, M. (2007). El diseño organizativo de la empresa familiar gallega. "El comportamiento de la empresa ante entornos dinámicos". XIX Congreso anual y XV Congreso Hispano-Francés de Asociación Europea de Dirección y Economía de Empresa (AEDEM), 1 (Ponencias), 61.

Tadeo Basco, R. (2005). Comportamiento en la dirección y gobierno de la empresa familiar: Análisis empírico de la profesionali204 zación como garantía de continuidad (Tesis Doctoral) España: Universidad Complutense de Madrid.

\footnotetext{
Cómo citar este capítulo:
}

Hernández-Fernández, L., Portillo-Medina, R., Hernández Chacín, A., y Romero Borré, J. (2017). La empresa familiar desde el sentir y el pensar: Un modelo de gestión del conocimiento. En Prieto-Pulido, R. y García-Guiliany, J. (2017). Gerencia de las organizaciones. Un enfoque empresarial. Ediciones Universidad Simón Bolívar. Barranquilla-Colombia. 183-204. 